\title{
Voluntariado en medicina. Proyecciones asociadas a su desarrollo
}

\author{
Rolle $\mathrm{A}^{1^{*}}$, Lubi $\mathrm{F}^{2}$, Ramirez $\mathrm{M}^{2}$
}

Resumen: Introducción: El voluntariado en Chile existe a múltiples niveles, destacando en este artículo los proyectos universitarios en que participan alumnos de medicina UC, dada su relevancia a nivel de formación extracurricular y en el proceso de adquisición de destrezas no técnicas relevantes en torno al profesionalismo médico. Esto ocurre tanto en relación con el trabajo logístico organizativo detrás de proyectos de esta índole, como en la transmisión de ideas-fuerza específicas entre quienes participan de estos como voluntarios. Sin embargo, no se conoce con detalle la participación de estudiantes en proyectos de voluntariado, ni su percepción respecto a estos. Objetivo: Este estudio busca describir la participación de alumnos de Medicina UC en proyectos de voluntariado, especialmente en relación con la Dirección de Pastoral y Cultura Cristiana UC. Método: Estudio descriptivo. Se utilizaron datos de participación en proyectos de la Dirección de Pastoral y Cultura Cristiana UC (años 2010-2013); se realizó una encuesta electrónica a estudiantes de Medicina, evaluando participación en voluntariado y otras actividades extracurriculares. Resultados: En los dos proyectos analizados en este estudio, (Misión País y Misión de vida) se observa que un alto porcentaje de los alumnos que participan lo hacen en cargos de liderazgo, que fluctúa en torno al 50\% en los diversos años y proyectos. La encuesta sobre actividades extracurriculares fue respondida por el 11,4\% de los encuestados. Entre ellos, se observó una alta participación en actividades de voluntariado (70,1\% ha participado en trabajos o misiones voluntarias durante algún verano de su formación universitaria), especialmente en relación con cargos específicos de liderazgo de proyectos. Conclusiones: Las actividades de voluntariado entre los estudiantes de Medicina constituyen una oportunidad muy relevante de formación de destrezas no técnicas, especialmente en habilidades de liderazgo, colaboración y trabajo en equipo. Futuras líneas de trabajo y proyecciones de estudio pueden incluir consolidar un buen catastro y estudios de seguimiento, así como analizar el impacto en la práctica clínica y en el desarrollo profesional y personal de la participación en proyectos de voluntariado.

Palabras clave: Destrezas no técnicas; educación médica; actividades extracurriculares.

Abstract: Background: Activities related to volunteering are widely presented in the university context, specially between students from the School of Medicine, Pontificia Universidad Católica de Chile. These activities are relevant towards an extracurricular development of non-technical skills related to medical professionalism. This is related to the organizational work needed to account the relevant tasks, and to the mission of each specific activity of volunteering. However, we don't know the perception of the students regards these activities, nor the details of the participation in each activity. Objective: To describe the participation of medicine students from the Pontificia Universidad Católica de Chile in volunteering projects. Special emphasis is done in projects related to the Dirección de Pastoral y Cultura Cristiana UC. Methods: Descriptive study. Data from participation in projects related to the Dirección de Pastoral de Cultura Cristiana UC were obtained for years 2010-2013. Also, an electronic survey was done to medicine students, evaluating participation in volunteering activities and in other extracurricular activities. Results: In both projects of volunteering analyzed in this study, there is a high participation of medical students between leadership positions. $11.4 \%$ of the medicine students competed the electronic survey. There is a high participation in volunteering activities, especially in relation to leadership positions. Conclusions: Between medicine students, volunteering activities are related to an opportunity of development of non-technical skills, specially related to leadership and teamwork. New studies could deepen into these activities, analyzing the potential impact in the clinical practice and the human and professional development of these students.

Keywords: Non-technical skills, medical education, extracurricular activities.

Fecha de envío: 19 de Agosto de 2016 - Fecha de aceptación: 4 de Diciembre de 2016

(1) División de Anestesiología, Facultad de Medicina, Pontificia Universidad Católica de Chile

(2) Escuela de Medicina, Pontificia Universidad Católica de Chile

Autor de correspondencia: ajrolle@uc.cl. 


\section{Introducción}

Las habilidades blandas son parte fundante de las destrezas requeridas por los profesionales de diversas carreras, en especial en el ámbito de la Medicina, donde incluso encontramos guías específicas de desarrollo según distintas especialidades (Gjeraa et al., 2016). Son atributos asociados al éxito en la obtención de metas, tanto a nivel académico como profesional. Entre las competencias a las que tradicionalmente este término se refiere, encontramos habilidades asociadas a la comunicación efectiva, liderazgo, organización y trabajo en equipo, empatía, profesionalismo y optimismo, entre muchas a desarrollar (de Ridder et al., 2014). El término "habilidades blandas", sin embargo, en la actualidad ha sido reemplazado por el concepto de "destrezas no técnicas". Se definen como el conjunto de habilidades o destrezas sociales, personales y cognitivas que, en complemento a las habilidades técnicas, contribuyen al cuidado seguro y eficiente de un paciente o de una situación en particular. De esta manera, el concepto viene a ser una evolución de las ideas relacionadas con las habilidades blandas, con una base conceptual similar (Flin et al., 2008). Operacionalmente, se pueden dividir en 7 categorías, siendo estas la conciencia situacional, comunicación, toma de decisiones, trabajo en equipo, liderazgo, manejo y cumplimiento de tareas, y la capacidad de sobrellevar el estrés y la fatiga (Flin \& Maran, 2015).

Estas destrezas son fundamentales en la práctica clínica, y pueden hacerse parte del proceso activo de aprendizaje de estudiantes del área de la Salud (Gade \& Chari, 2013). Pueden aprenderse de manera conceptual durante la formación profesional, para ser ejecutadas posteriormente en la práctica clínica, o se pueden obtener de manera indirecta, sin una enseñanza formal, en instancias curriculares y extracurriculares. Es posible enseñar y modelar, en cierto grado, el desarrollo de estas competencias, pese a dificultades en la estandarización de la evaluación (Lazarus, 2013). En asociación a actividades curriculares, por ejemplo, destaca la observación de la interacción de los profesionales médicos, o del área de la Salud, con sus pares y con los pacientes a su cargo, donde se desarrollan situaciones de liderazgo, comunicación efectiva y empatía, en el contexto de la práctica clínica. De las actividades extracurriculares asociadas a formación de destrezas no técnicas, destacan las actividades de voluntariado, en las cuales el desarrollo de estas mismas se da entre pares, con un objetivo en común a cumplir, sea o no asociado al ámbito de la Medicina.

En relación con la definición de voluntariado, una aproximación válida que cubre los aspectos esenciales podría ser la de actividad no remunerada, en la que una o más personas fortalecen una comunidad específica, con el fin de compartir y colaborar en el desarrollo de esta, sea por medio de construcciones para la comunidad o por espacios de encuentro. Puede tener objetivos específicos asociados al crecimiento personal de los voluntarios, por ejemplo, desarrollando habilidades blandas o planteando inquietudes asociadas a la actividad, extrapolables a la vida cotidiana. Las alternativas respecto a qué es lo que se realiza en concreto en instancias de voluntariado son múltiples, no excluyentes unas de otras. Construcciones en espacios comunes, casas básicas para familias, reforzamiento escolar, apostolado religioso y talleres de desarrollo para la comunidad según necesidades percibidas, son algunos de los caminos utilizados según los objetivos específicos que busque cada actividad. Se desarrollan en general a partir de la realidad del país, con un diagnóstico específico de necesidades a resolver. Según los resultados iniciales, las solicitudes específicas que realicen las comunidades apoyadas y el aporte de los mismos voluntarios, el proyecto se desarrolla para cumplir su misión de manera integral y completa. El objetivo asociado, en relación con el crecimiento personal de quienes participen, se presentará de manera más o menos patente según cada proyecto, siendo fundamental en ese sentido el énfasis que muestren en ello quienes dirijan el desarrollo de las actividades de voluntariado.

\section{Objetivo}

Describir la participación de alumnos de Medicina UC en diversos proyectos de voluntariado. Se pone énfasis en proyectos relacionados con la Dirección de Pastoral y Cultura Cristiana UC, dada la existencia de registros fidedignos de participación a lo largo de varios años.

\section{Metodología}

Estudio de diseño observacional, y carácter transversal. Se solicitaron datos relacionados con la participación de estudiantes de Medicina UC en dos proyectos relacionados con la Dirección de Pastoral y Cultura Cristiana UC, entre los años 2010 y 2013..En paralelo, se realizó una encuesta electrónica a estudiantes de Pregrado de la Escuela de Medicina de la Pontificia Universidad Católica de Chile, cursando estudios durante noviembre del año 2015 (número de alumnos en esa situación: 840 ). Se consultó respecto a la participación (actual o pasada, durante el período universitario) en diversas actividades extracurriculares, en el contexto de representación estudiantil, deportes o acción social, sea en trabajos o misiones, durante el año o en período de vacaciones. Dichas respuestas no fueron excluyentes entre sí, y se presentan en la Tabla 1.

\section{Resultados}

La participación de estudiantes del área de Medicina en proyectos de la Dirección de Pastoral y Cultura Cristiana UC, se da tanto como parte de la organización de proyectos, en diversos cargos de 
liderazgo y logística, como en participación durante el desarrollo de estos sin tareas específicas. En la Figura 1, se presenta participación entre los años 2010 y 2013 en los proyectos Misión País y Misión de Vida, y la relación de cargos específicos de trabajo en estos proyectos, respecto a voluntarios. Tomando datos de Misión País 2013, proyecto de la Universidad Católica que lleva más de 10 años al servicio de la evangelización por Chile, se consignan 24 participantes de la Escuela de Medicina, de los cuales el 50\% participó con cargos de liderazgo y organización, ya sea en las diversas comunidades desarrolladas o en el marco del proyecto en su globalidad. Esto corresponde a un 2,8\% de los estudiantes de Medicina, participando en uno solo de los proyectos disponibles en nuestra Universidad. En los últimos 5 años, los datos de participación para este mismo proyecto varían entre 2,38\% y 2,97\%, con participación en cargos de liderazgo que fluyen entre $36,4 \%$ y $55 \%$. En proyectos con menor número de participantes, como es el caso de Misión de Vida UC, estas diferencias en cargos de participación son aún más notorias, alcanzando el 66,6\% de cargos de liderazgo, entre el total de estudiantes de Medicina UC asistentes al proyecto. Más datos al respecto se encuentran en la Figura 1.

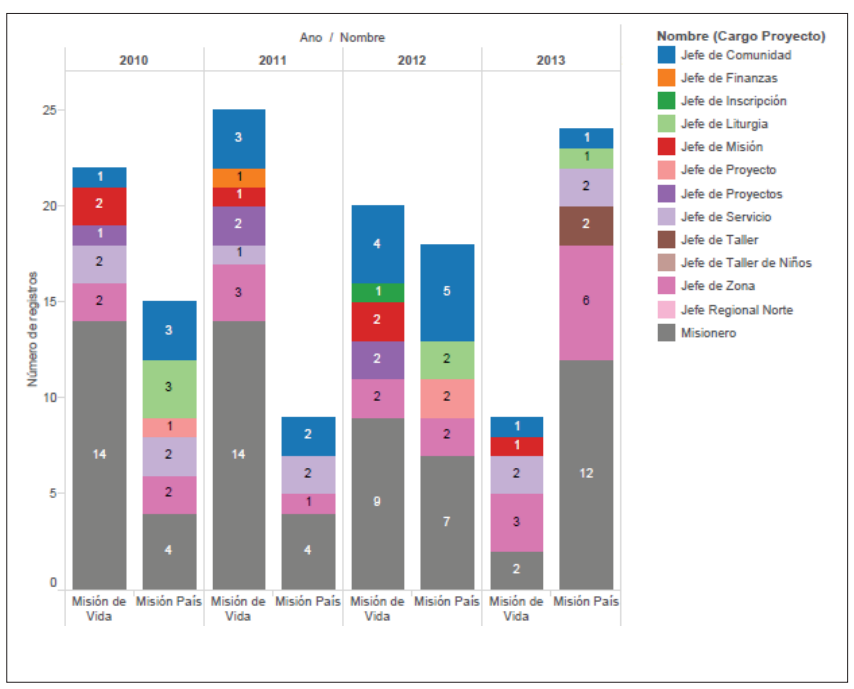

Figura 1. Participantes en proyectos Misión País y Misión de Vida UC, años 2010-2013, desglosados según actividad realizada. En gris, los participantes sin cargos específicos en el diseño o implementación de los proyectos. En colores, los participantes con diversos cargos de liderazgo en los proyectos.

La encuesta fue respondida por 97 estudiantes, correspondientes al $11,4 \%$ de la población. Un 70,1\% afirmó haber participado de trabajos o misiones voluntarias en período de vacaciones, siendo la actividad más mencionada. Trabajos o misiones durante fines de semana, fueron la segunda actividad más mencionada, con un $57,7 \%$ de las respuestas. En tercer lugar, se mencionó a las actividades deportivas con entrenamiento semanal, por un $55,7 \%$ de los encuestados.
Un 82,5\% de los encuestados, respondió haber participado al menos en 2 de los ítems mencionados, incluidos en la Tabla 1.

Tabla 1. Respuestas posibles ante la pregunta de selección múltiple ¿Has participado de alguna de estas actividades extracurriculares algún año durante el período universitario? Se permitió marcar más de 1 respuesta.

\begin{tabular}{l}
\hline Centro de Estudiantes \\
\hline Representación Estudiantil \\
\hline Trabajos/misiones voluntarias en invierno/verano \\
\hline Trabajos/misiones voluntarias durante los fines de semana de un \\
semestre \\
\hline Reforzamiento escolar \\
\hline Actividades deportivas, con entrenamiento semanal \\
\hline Actividades de Pastoral Universitaria/Extrauniversitaria \\
\hline Otros
\end{tabular}

\section{Conclusiones}

En los proyectos Misión País y Misión de Vida, de la Dirección de Pastoral y cultura cristiana, destaca el hecho de que los alumnos de medicina tienden a tomar cargos de liderazgo en un porcentaje superior al 50\%. El número de participantes en un momento fijo en Misión país (noviembre 2015) es de alrededor de un 2,8\% del total de alumnos de la Escuela. Entre las actividades extracurriculares de la Escuela de Medicina -medidas por la encuesta-, destacan las misiones y trabajos voluntarios, sea durante vacaciones o en fines de semana, como las actividades más mencionadas por los estudiantes. Se debe considerar que la encuesta fue respondida por el 11,4\% del universo de estudiantes de Medicina UC, por lo que no se puede asumir que sea una muestra representativa y es posible que las respuestas hayan sido precisamente de quienes más compromiso sentían respecto a estas actividades.

Las actividades asociadas al desarrollo humano integral son parte fundamental de toda carrera universitaria (Lazarus, 2013), en especial en aquellas asociadas al área de la Salud, dada la constante relación con personas durante la práctica profesional y clínica. Destacan en este sentido las destrezas no técnicas, que proporcionan herramientas para desenvolverse en el ámbito profesional y humano con buenas relaciones interpersonales, con el fin de trazar metas en común y dirigirse a ellas como equipo, tratando a cada persona, y a cada paciente, con la dignidad humana que merecen. En este escenario, su desarrollo, tanto por medio de actividades curriculares como extracurriculares, se imponen como un desafío para toda instancia formadora de profesionales, con particular relevancia en aquellos asociados al área de la Salud. Las actividades de voluntariado, tanto para quienes las organizan como para quienes participan de ellas, pueden cubrir parte de esta necesidad, gracias a los múltiples proyectos existentes, según intereses personales y objetivos específicos 
que presentan. El fortalecimiento y crecimiento de instancias de este tipo, debe plantearse entre quienes participen de procesos de formación de profesionales a mediano plazo.

Entre las falencias encontradas en relación con el desarrollo del voluntariado en nuestro país, y en nuestra Universidad, se presenta el problema de no existir un catastro formal de todos los proyectos de participación estudiantil, abierta a todo público. Esto impide el seguimiento de los proyectos a nivel global. No existen, así mismo, estudios respecto al desarrollo universitario y profesional en relación a la participación de proyectos de voluntariado ni del grado de conocimiento de estos espacios por parte de estudiantes de Medicina.

La Universidad Católica, por medio de proyectos de voluntariado en funcionamiento actualmente, entrega herramientas para el desarrollo de destrezas no técnicas entre sus estudiantes, tanto del área de la Salud como externos a esta. La participación de estudiantes de Medicina en liderazgo de proyectos de voluntariado es un tema que debe ser medido de manera más transversal y constante, con estadísticas actualizadas y que consideren los diversos proyectos existentes en la Universidad.

Futuras líneas de trabajo y proyecciones de estudio pueden incluir el impacto en la práctica clínica de la participación de estudiantes de Medicina en proyectos de voluntariado, y la percepción de desarrollo personal en relación a la participación en estos. Otras áreas de estudio pueden asociarse al grado de conocimiento de los estudiantes de Medicina respecto a la relevancia del desarrollo de destrezas no técnicas, y de la identificación de espacios curriculares y extracurriculares disponibles para la formación profesional en este ámbito.

\section{Referencias}

de Ridder J, Meysman P, Oluwagbemi O \& AbeelT. (2014). Soft skills: an important asset acquired from organizing regional student group activities. PLoS computational biology 10, e1003708.

Flin R \& Maran N. (2015). Basic concepts for crew resource management and non-technical skills. Best practice \& research Clinical anaesthesiology 29, 27-39.

Flin RH, O'Connor P \& Crichton M. (2008). Safety at the Sharp End: A Guide to Non-technical Skills. Ashgate.

Gade S \& Chari S. (2013). Case-based learning in endocrine physiology: an approach toward self-directed learning and the development of soft skills in medical students. Advances in physiology education 37, 356-360.

Gjeraa K, Jepsen RM, Rewers M, Ostergaard D \& Dieckmann P. (2016). Exploring the relationship between anaesthesiologists' non-technical and technical skills. Acta anaesthesiologica Scandinavica 60, 36-47.

Lazarus A. (2013). Soften up: the importance of soft skills for job success. Physician executive 39, 40-45. 